\title{
THERMODYNAMICS OF IRREVERSIBLE PLANT CELL GROWTH
}

\author{
MARIUSZ PIETRUSZKA, SYLWIA LEWICKA, \\ KRYSTYNA PAZURKIEWICZ-KOCOT \\ Faculty of Biology and Environmental Protection, University of Silesia \\ Jagiellońska 28, 40-032 Katowice, Poland \\ e-mail: pietruma@us.edu.pl
}

(Received: October 17, 2005. Accepted: December 1, 2005)

\begin{abstract}
The time-irreversible cell enlargement of plant cells at a constant temperature results from two independent physical processes, e.g. water absorption and cell wall yielding. In such a model cell growth starts with reduction in wall stress because of irreversible extension of the wall. The water absorption and physical expansion are spontaneous consequences of this initial modification of the cell wall (the juvenile cell vacuolate, takes up water and expands). In this model the irreversible aspect of growth arises from the extension of the cell wall. Such theory expressed quantitatively by time-dependent growth equation was elaborated by Lockhart in the 60's. The growth equation omit however a very important factor, namely the environmental temperature at which the plant cells grow. In this paper we put forward a simple phenomenological model which introduces into the growth equation the notion of temperature. Moreover, we introduce into the modified growth equation the possible influence of external growth stimulator or inhibitor (phytohormones or abiotic factors). In the presence of such external perturbations two possible theoretical solutions have been found: the linear reaction to the application of growth hormones/abiotic factors and the non-linear one. Both solutions reflect and predict two different experimental conditions, respectively (growth at constant or increasing concentration of stimulator/inhibitor). The non-linear solution reflects a common situation interesting from an environmental pollution point of view e.g. the influence of increasing (with time) concentration of toxins on plant growth. Having obtained temperature modified growth equations we can draw further qualitative and, especially, quantitative conclusions about the mechanical properties of the cell wall itself. This also concerns a new and interesting result obtained in our model: We have calculated the magnitude of the cell wall yielding coefficient $\Phi(T)\left[\mathrm{m}^{3} \mathrm{~J}^{-1} \cdot \mathrm{s}^{-1}\right]$ in function of temperature which has acquired reasonable numerical value throughout.
\end{abstract}

KEY WORDS: cell wall yielding, growth stimulators/inhibitors, modified growth equations.

\section{INTRODUCTION}

One of the most complex physiological processes in plants is growth (Fogg 1975; Kutschera 2000 and papers cited therein, see for a review). This process is based on irreversible extension of the whole organism due to the increase of the quantity and size of cells, the mass of protoplast and the cell walls. In the growth of arbitrary organ one can differentiate three basic phases: the initial phase of slow growth, the intense growth phase and, eventually, the final phase of slow growth. Such regularity which can be represented by sigmoid curve is known as the law of great growth ("S"-shaped curve). The sigmoid curve characterizes the course of individual cell growth, plant organs and the growth of plant as a whole. The dominating length increments in the time unit are observed approximately at the mid-time of growth. The period of intense growth lasts fairy short and depends on plant species.
The plant growth is influenced by physical and biotic factors of environment (Wright 1966; Trewavas 1991; Edelmann 1995). The external factors which fundamentally influence on plant growth are: temperature, light, water and soil factors, and atmosphere composition. The dependence of growth on temperature, which is similar to the another physiological processes, is described by van't Hoff law. One can distinguish the minimum temperature (below which the growth is inhibited), the optimal temperature (the most intense growing rate) and the maximum temperature (the growth is no more possible). In the limits of temperature minimum or optimum when the only factor limiting physiological process (e.g. growth) is temperature then, according to the van't Hoff law, the intensity of such process is doubled at temperature increase by 10 degrees Celsius (logarithmic dependence).

The growth dependence on temperature indicates the fact that with the temperature elevation the intensity of the 
growth process gradually increases (which is also due to the acceleration of chemical reactions by raising temperature). However, after exceeding the optimum temperature the rapid decrease of the intensity of plant growth starts. The experimental relations between temperature, turgor pressure and the growth of plant cells were thoroughly investigated in paper by Proseus et al. (2000).

On the other hand, regarding different heat requirements one can divide plants on criophyties (growth optimum phase beneath $20^{\circ} \mathrm{C}$ ), mesophyties (optimum growth rate at $20-40^{\circ} \mathrm{C}$ ) and thermophyties which grow most intensely above $40^{\circ} \mathrm{C}$. In the above context another division line is connected with plant species and organs, growth phase and with the age of individual plant.

In spite of external factors one should also mention that growth, as being complex, depends on many physiological processes (internal factors) like respiration and photosynthesis as well as on enzymes activity, including $\mathrm{H}^{+}$ATPase localized in plasmalemma.

The tale of Jack and the Beanstalk includes an episode in which bean seedlings grow up high through the clouds in a single night. Such event rises questions. What limits the growth rate? What is the role of temperature in the growth rate of real plants? The first question is broadly discussed in the review article (Cosgrove 1986) where the prevailing concept of the plant cell growth results from turgor-driven yielding of the cell wall which is a physical description of how plant cells increase in size during growth and morphogenesis. In this context two questions are raised (a) about the characteristics which enable the cell wall to extend, and (b) how is water transported to and absorbed by growing cells to maintain turgor pressure while prolonged extension of the cell wall. The above questions raised by Cosgrove (1986) in his review put emphasis on the most recent papers on physical constraints which limit plant growth. Due to the author's opinion the irreversible enlargement of plant cells results from two independent physical processes. The first one is water absorption which increases the volume of growing cells consisted mainly of $85-95 \%$ of water. The second is the wall extension which generates the driving force for the water uptake.

Besides the factors of external nature the growth regulators are of the fundamental importance in growth and development, see also Cleland (1986). These substances stimulate or inhibit the processes of growth. The regulators of growth, applied in physiological concentrations, which stimulate the plant growth are called plant growth stimulators and, in the contrary, the substances, adapted also in such physiological concentrations, which inhibit growth are defined as plant growth inhibitors. The natural growth regulators (plant phytohormones) include auxins, giberellins, cytokinins, the abscisin acid and ethylene.

The goal of the present paper is to introduce, after reasonable precautions and discussion, the notion of temperature to the growth equations via the state equation ${ }^{1}$. This enable us to create a new phenomenological (thermodynamic) model of the temperature influenced growth. After receiving appropriate equations we adjust these to the experimental

${ }^{1}$ Despite some obvious, however fully justified, assumptions and simplifications the authors' intention was to obtain analytic solutions which yield clear interpretations. Any other approach would result in numerical calculations. data and find quantitative description of the elongation of maize versus time, parameterized by temperature. Two major solutions of these equations are found: (a) the linear solution for unperturbed growth, (b) linear and non-linear solutions of growth influenced by external perturbation (growth hormones or abiotic factors), which in fact both reflect different conditions of experiment. Moreover, we notice that the foregoing major consequence of our model is the new possibility to provide a good working mathematical description of the most essential features of physical properties of the cell wall (regarding wall yielding in function both on time and temperature). Despite the fact that our paper makes intense use of mathematics an effort is made to make our article intelligible to all experimental biologists, especially plant physiologists (for this purpose the main body of our paper does not contain punctilious derivations, however we move the detailed step by step calculations into the Appendix A). We also expect that our theory will give reproducible results regarding different plant species (in comparative studies) and give direct, simple and comprehensive method for quantitative predictions (calculations) for plant physiologists who can draw further conclusions about all these properties which like the wall yielding depend on temperature.

After the above outlined general Introduction into the problems of plant growth in the context of temperature, in the following Section we comment details of the authors' experiment. In the next Section, which is the central part of our paper, we briefly introduce the physical model of temperature affected plant cell growth which next we discuss in the following Subsection in both cases of unperturbed and perturbed growth. In the light of equations of our model the experimental results are briefly outlined. Also the main theoretical outcome is discussed: the extensibility of the cell wall in function of temperature. In the last Section we present the final remarks. Step by step derivations of our equations in the above mentioned three different cases are presented in the Appendix A. Detailed discussion about the foundations of our model is presented in the Appendix B.

\section{MATERIAL AND METHODS}

The experiments were carried out with four day old maize plants (Zea mays L.) grown on Hoagland's medium (Hoagland and Arnon 1950) at $27^{\circ} \mathrm{C}$. Seeds of maize were cultivated in darkness. Then individual segments were transferred to an aerated solution containing standard micro- and macro-elements. Two kinds of experiment were performed.

(a) Unperturbed experiment: The coleoptile segments were divided into eight groups growing at different temperatures from $5^{\circ} \mathrm{C}$, increased by $5^{\circ} \mathrm{C}$ up to $40^{\circ} \mathrm{C}$. Each group was represented by 10 segments. The experiment was carried out within 7 hours. The measurements were taken at every 1 hour. The individual values presented in Figure 1 are the averages obtained from 10 measurements.

(b) Perturbation present: The coleoptile segments were divided into three groups growing at temperatures 25,30 and $35^{\circ} \mathrm{C}$, respectively. The experiment was carried out within 8 hours, and the measurements were taken at every 1 hour. (i) Linear response case: As a stimulator the auxin in physiological concentration $10^{-5}$ mole was applied after 4 


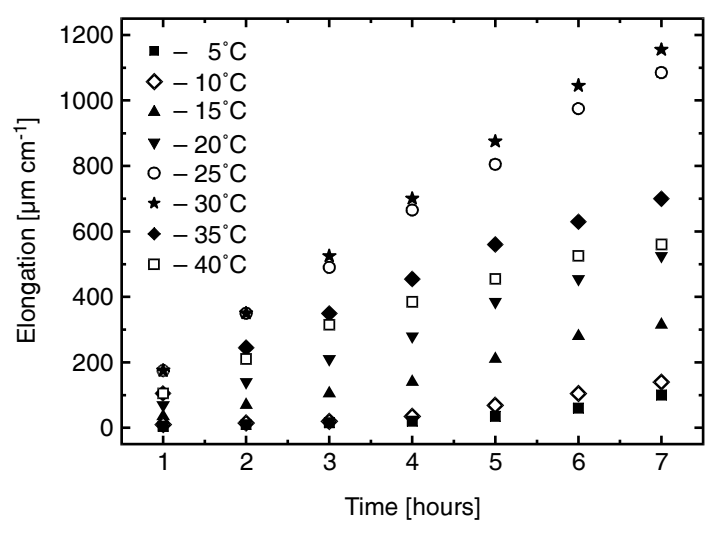

Fig. 1. Experimental results: Nonperturbed elongation of maize (Zea mays L.) coleoptile segments versus time at eight different temperatures. Linear behaviour is clearly visible.

hours of unperturbed growth; as an inhibitor we introduced $\mathrm{CdCl}_{2}\left(10^{-5}\right.$ mole) also after 4 hours (see Fig. 2). (ii) Nonlinear response case: As a stimulator the auxin was applied after 4 hours $\left(1.25 \cdot 10^{-6}\right.$ mole) of unperturbed growth, then after 5 hours $\left(2.5 \cdot 10^{-6}\right.$ mole), after 6 hours $\left(5 \cdot 10^{-6}\right.$ mole $)$ and after 7 hours $\left(10^{-5}\right.$ mole); as an inhibitor $\mathrm{CdCl}_{2}$ was applied after 4 hours $\left(1.25 \cdot 10^{-6} \mathrm{~mole}\right)$ of unperturbed growth, then successively after 5 hours $\left(2.5 \cdot 10^{-6}\right.$ mole $), 6$ hours $\left(5 \cdot 10^{-6}\right.$ mole $)$ and after 7 hours $\left(10^{-5} \mathrm{~mole}\right)$, see Figure 3.

The individual values presented in Figures 2 and 3 are the averages obtained from 5 measurements. In all experimental cases the control measurement was performed. In all experiments the elongation was measured by microscope. The relative error was of about $5 \%$.

\section{TEMPERATURE MODIFIED GROWTH EQUATIONS}

In the model of plant cell growth the cell turgor pressure remains in dynamic balance between wall extension which tends to dissipate turgor pressure and water uptake acting to restore it (Cosgrove 1986). Lockhart (1965) proposed a simple (time-dependent) differential equation

$$
\frac{1}{V} \frac{d V}{d t}=\Phi(P-Y)
$$

which states that the relative growth rate (cell volume $-V$ ) depends linearly on hydrostatic pressure $(P)$ in excess of a critical turgor $(Y)$ linked by wall extensibility coefficient $(\Phi)$. In this model of growth, turgor pressure coordinates water uptake with cell wall extension. However, plants do not grow in a space which is disconnected to the real world. They usually grow in some special conditions where one of the most important growth factors is temperature. In order to solve the problem in our approach we part, as usual in thermodynamics, the whole system into the investigated sample (here: plant cell) and 'the rest of the world' - thermostat (the environment) which remains at a constant temperature $T$ (see Fig. 4). Aiming to bring in temperature into the above equation we utilize the state equation ${ }^{2}$

\footnotetext{
${ }^{2}$ Here, in order to justify and give foundations to our approach, we provide the following comment (for full discussion see also the Appendix
}
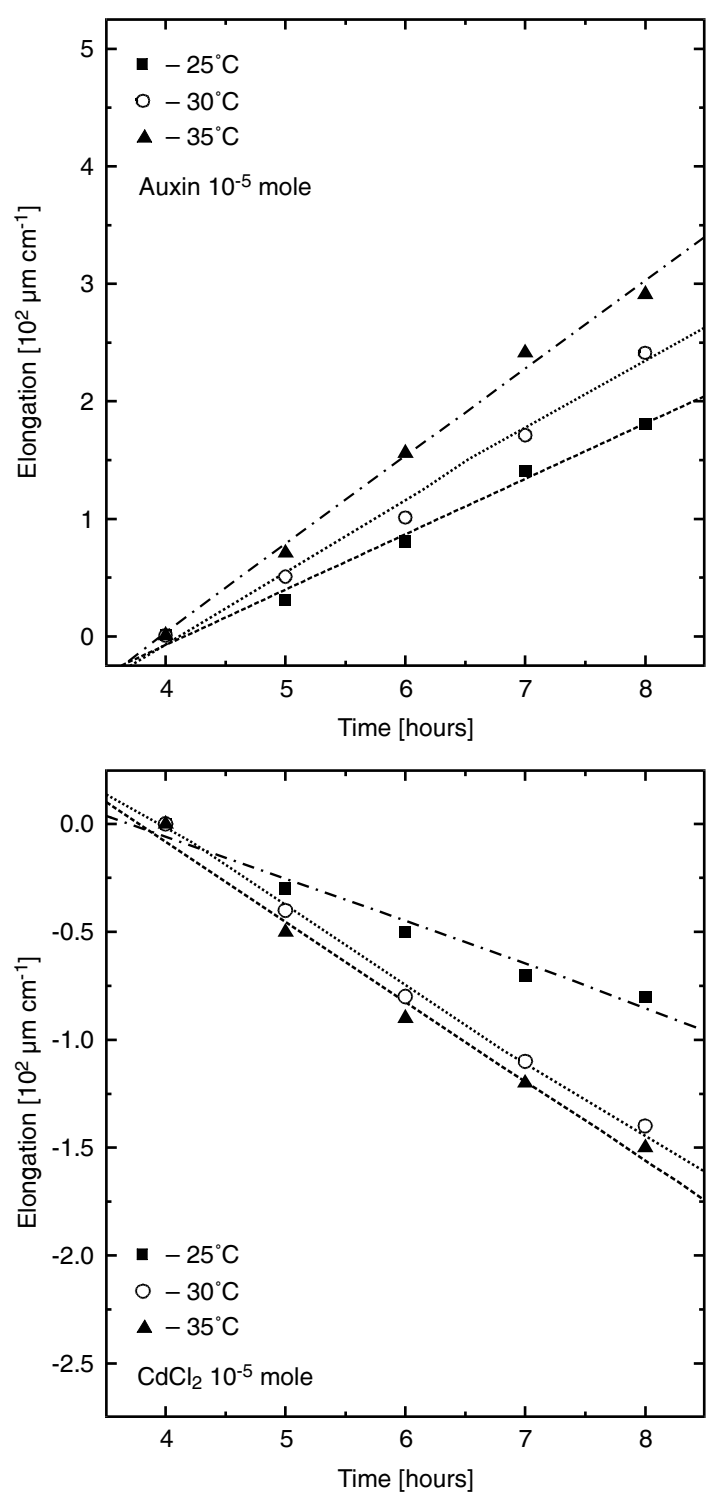

Fig. 2. Experimental results: Perturbed elongation (the linear response) of maize (Zea mays L.) coleoptile segments versus time at three different temperatures for both stimulator and inhibitor applied after 4 hours. The values of control growth have been subtracted.

B). In thermodynamics, an equation of state is a constitutive equation describing the state of matter under a given set of physical conditions. Especially, it delivers a mathematical relationship between two or more state functions associated with the matter, such as its temperature, pressure, volume, or internal energy. The most prominent use is to predict the state of matter (e.g. ideal gas law, van der Waals equation). One of the simplest equations of state for our purpose is the ideal gas law (roughly accurate for gases at low pressures and high temperatures) which should obviously be treated heuristically (not literally) in this case. However, the use of this equation, is fully justified in the $1^{\text {st }}$ approximation if one begins with the virial theorem and restrict only to the first term of expansion. Moreover, equivalently the same result we acquire, when we assume the properties of water (very weak compressibility) and expand the resulting exponent to the linear term in temperature $T$. However, if appropriate assumptions are made about the mathematical form of intermolecular forces in the water solution filling up the plant cell interior, higher coefficients in the virial expansion should be included. For the sake of clarity of the presented theory we restrict ourselves to the term linear in $T$. 

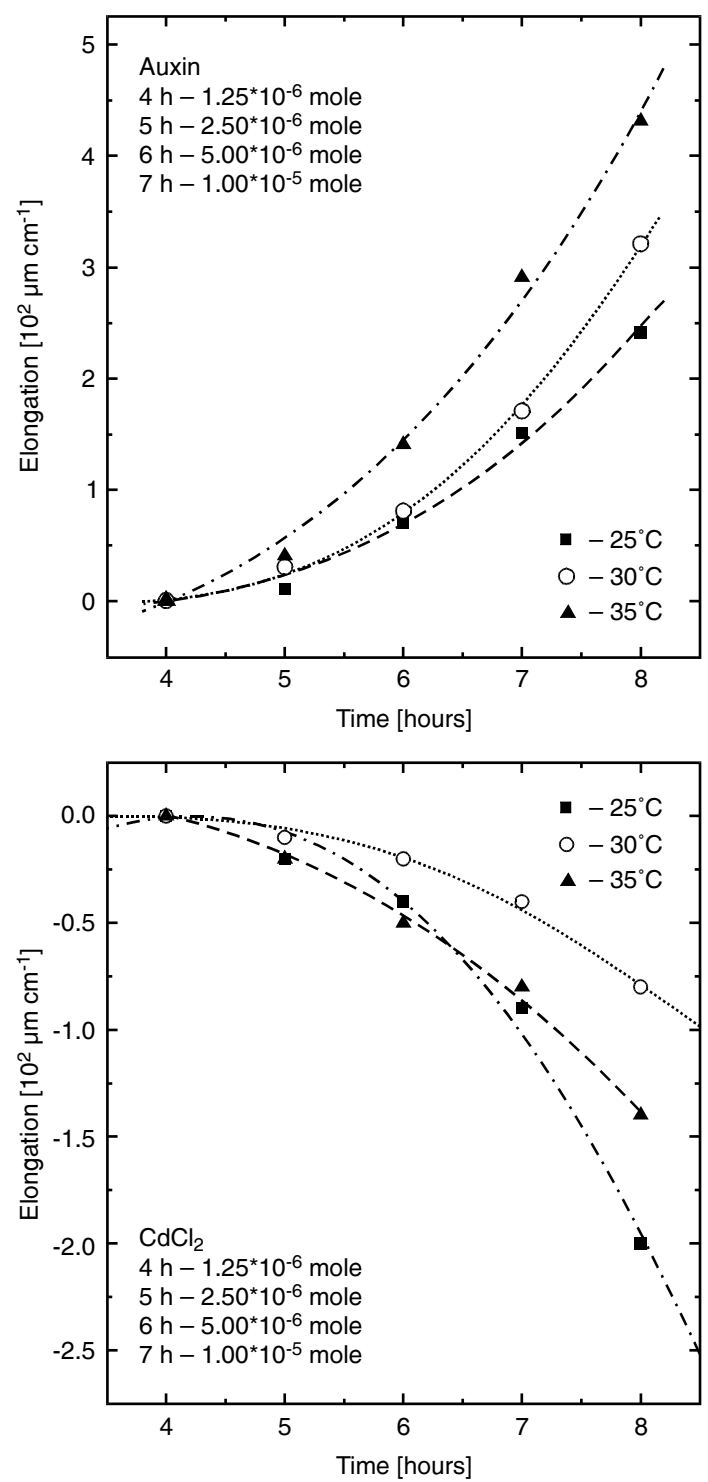

Fig. 3. Experimental results: Perturbed elongation (the non-linear response) of maize (Zea mays L.) coleoptile segments versus time at three different temperatures. The "continuous" concentration change starts after 4 hours from the beginning of the experiment. The values of control growth have been subtracted.

$P(T, V)=n \mathrm{R} T / V$ where $T$ is the absolute temperature (in Kelvin scale), and $V$ is proportional to the amount of water in the cell $(\approx V) ; n$ is the number of water moles, $\mathrm{R}-$ the universal constant (in our phenomenological model the movement of the piston reflects the extensibility properties of the cell wall, see Figure 4) ${ }^{3}$. Accordingly, we wish to solve the following equation

${ }^{3}$ By introducing our physical model we are far beyond the simplified picture where we interpret the movement of a piston as only reflecting the compressibility/extensibility properties of the water solution inside the plant cell. In contrary, in such a way we incorporate rather a number of basic chemical and biochemical processes which accelerate or inhibit the growth in function of temperature (kinetics of chemical reactions, metabolism, photosynthesis (biomass production), protein denaturing, etc.). Because both type of processes act simultaneously, however with different intensity at distinct temperature ranges, one should expect a crosso-

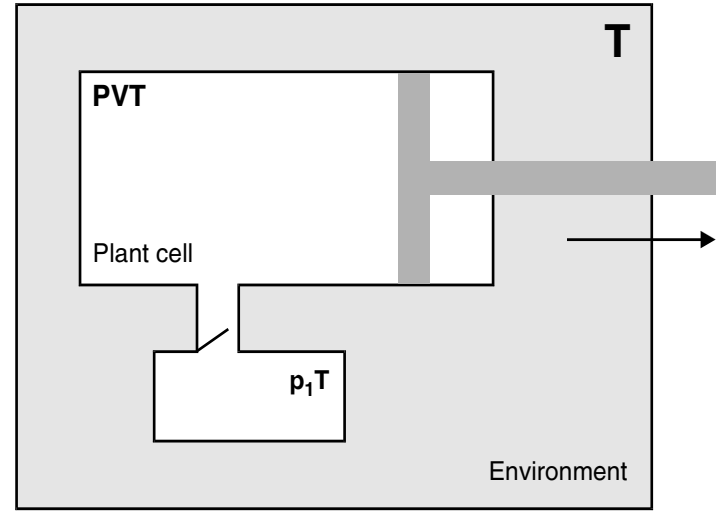

Fig. 4. Scheme of the 'Gedanken experiment' set-up: The movement of the piston in the cylinder (plant cell) reflects the extensibility properties of cell wall. The additional container with pressure $\mathrm{p}_{1}$ and the valve is bound with action of a stimulator or an inhibitor which can be opened at a time $t_{0}<t=t_{1}$ to release pressure $\mathrm{p}_{1}$. The whole system is immersed into thermostat (environment) at temperature $T$.

$$
\frac{1}{V} \frac{d V}{d t}=\Phi(T)(P(T, V)-Y(T, V))
$$

which after inserting $Y=a T / V, a>0$ (this assumption seems to be fully justified since $Y$ has the same character as $P$ ), can be rewritten as

$$
\frac{1}{V} \frac{d V}{d t}=\Phi(T)\left(n R \frac{T}{V}-\frac{a T}{V}\right)
$$

where in contrary to Eq. (1) the coefficient $\Phi=\Phi(T)$ (see for discussion the Appendix B). By substituting $\gamma \equiv n \mathrm{R}-a$ we get the following equation

$$
\frac{1}{V} \frac{d V}{d t}=\Phi(T)(n R-a) \frac{T}{V}=\Phi(T) \gamma \frac{T}{V} .
$$

A simple calculus (see Appendix A, Case 0 for details) yields a general equation for the volume $V$ we have been looking for

$$
V=V_{0}+\underbrace{\Phi(T) \gamma T t}_{\text {slope }}
$$

which is linear in time $t\left(V_{0}=V\left(t=t_{0}\right)\right.$ stands for the initial volume of the cell). From the experimentalist' point of view the most interesting part is the slope which, as a whole, depends on temperature and can be acquired from the fit to the experimental data. However, what we measure in experiment is the elongation and the above equation holds

ver from one type of behavior to the other. Thus, there should always exist a specific, well defined critical temperature $\left(\tau^{*}-\right.$ in Celsius scale) for which the growth rate is optimal. Considering temperatures in Kelvin scale and multiplying $T^{*}$ by the Boltzmann constant $\left(\mathrm{k}_{\mathrm{B}} T^{*}\right)$ we gain also clear energetical interpretation bound with the peak energy absorption necessary for biological processes for the optimum growth. 


$$
\operatorname{Elong}(t) \approx \frac{V-V_{0}}{V_{0}}=\underbrace{\frac{1}{V_{0}} \Phi(T) \gamma T t}_{\text {slope }}=\frac{1}{V_{0}} \Phi(\tau) \gamma(\tau+273.15) t
$$

By having determined the values of the directional coefficient (slope) one can calculate the temperature dependence of $\Phi(\tau)$ and, consequently, draw conclusions about the behavior of the cell wall itself (see Fig. 5). Based on the linear fits (absolutely justified for evidently co-linear plots in Figure 1) to the unperturbed growth data we receive the set of 8 directional coefficients which in turn (through Eq. (6)) deliver the set of 8 values of $\Phi(\tau)$ for originally pre-set temperatures in the experiment. All these such calculated values are depicted in Figure 5. The dotted curve is obtained by the fit to the Lorentz-like distribution ${ }^{4}$

$$
\frac{\phi_{0} \tau}{\alpha^{2}+\left(\tau-\tau^{*}\right)^{2}}
$$

where $\phi_{0}$ and $\alpha$ stand for the height and the width of the Lorentz curve, respectively; $\tau^{*}$ denotes the calculated optimum (critical) temperature of growth, $\tau$ is a measured temperature (in Celsius scale) and $T=\tau+273.15^{\circ} \mathrm{C}$. An amazingly high determination coefficient occurs (see the legend in Fig. 5). Here, we stress that we have presented not only the qualitative picture of wall extension coefficient but the quantitative values of $\Phi$ are also calculated.

\section{EXTERNAL STIMULATIONS}

Moreover, as we have noticed in the introductory part of this paper, the plant growth can be stimulated or inhibited by phytohormones or abiotic factors, respectively. The latter, however, can be also applied in a certain period of time after incubation, say at a time $t=t_{1}$ (the experiment starts at $t_{0}$ ). From the mathematical point of view it means (see also Fig. 4) that we perform the following transformation:

$$
P(T, V) \rightarrow P(T, V)+p_{1} \theta\left(t-t_{1}\right)
$$

where the action of pressure $p_{1}<0$ can be interpreted as plant growth inhibitor, and of pressure $\mathrm{p}_{1}>0$ as stimulator. Such assumption will modify the central equation of our model (Eq. 2) in the following way (see also the Appendix A, Case 1):

$$
\frac{1}{V} \frac{d V}{d t}=\Phi(T)\left(\frac{\gamma T}{V}+p_{1} \theta\left(t-t_{1}\right)\right)
$$

\footnotetext{
${ }^{4}$ Let us justify the choice of such a function: The outlined system (plant cell) behaves similarly to the most systems described by a differential equation where both dissipative and extortive forces are present. In such systems there always exist a variable which is optimal at certain conditions (like resonance frequency $\omega=\omega^{*}$ for the harmonic oscillator). In our case the factor enforcing the crossover from accelerating to decelerating growth is temperature $\tau$. Analogically to the example with the harmonic oscillator, also in our case there must exist a critical ("resonance") temperature $\tau=\tau^{*}$. Consequently, the system should be described by a resonance curve - the Lorentz distribution function - in our case, however,
}

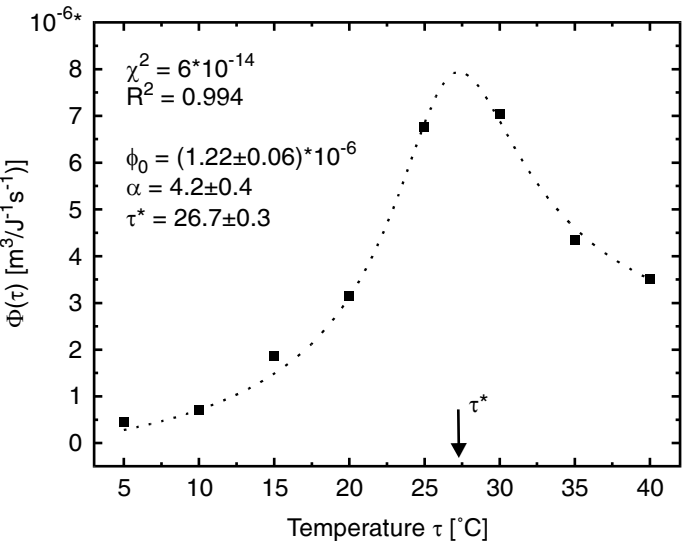

Fig. 5. The Lorentz-like fit to the calculated results for the unperturbed elongation of maize (Zea mays L.): theoretically predicted cell wall extensibility $\Phi(\tau)\left[\mathrm{m}^{3} \mathrm{~J}^{-1} \cdot \mathrm{s}^{-1}=\mathrm{m}^{2} \mathrm{~N}^{-1} \cdot \mathrm{s}^{-1}\right]$ versus temperature. $\tau^{*}$ points to the critical (optimal) temperature of the cell wall yielding

where $\theta\left(t-t_{1}\right)$ is the Heaviside theta step function acting as a trigger, and opening the valve of the container with pressure $\mathrm{p}_{1}$ at time $t_{1} ; \mathrm{p}_{1}=\mathrm{b}_{1} / V$, and $\mathrm{b}_{1}=$ const.

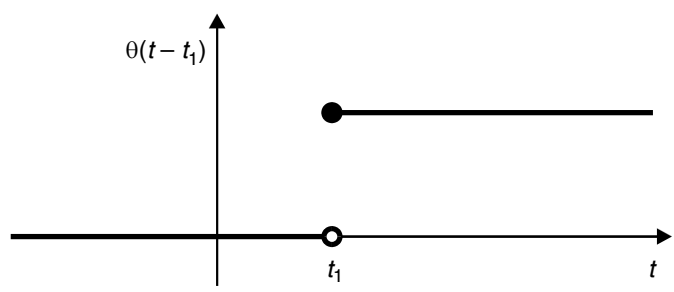

At this point we, again, face the problem of solving the differential equation (Eq. 8). After a simple calculus (we omit these steps here for brevity; see Appendix A, Case 1 for details) we arrive at

$$
V(t)=\left\{\begin{array}{cc}
V_{0}+\Phi \gamma T t & t<t_{1} \\
V_{0}+\Phi\left(\gamma T+b_{1}\left(t-t_{1}\right)\right) t & t \geq t_{1}
\end{array}\right.
$$

and $V_{0}=V\left(t=t_{0}\right)$, see also Appendix A, Eq. (14). In the above scenario, from the biological experiment point of view, we add only once at a time instant $t_{1}$ a droplet of a given growth stimulator or inhibitor (or equivalently we put our coleoptile segment into a solution containing growth stimulator/inhibitor at a given concentration after period $t_{1}$ $-t_{0}$ ). However, another possible outline can be thought of. Say, when we assume $\mathrm{p}_{1}=$ const., then we obtain inhomo-

modified by factor $\tau$. The latter modification is due to the fact that at $\tau=$ $0^{\circ} \mathrm{C}$ the growth must cease altogether. 
geneous differential equation which can be solved by means of constant variation (such procedure is briefly outlined in the Appendix A, Case 2). This scenario reflects the experimental situation when we constantly add into the environment the solution of a given growth stimulator or inhibitor after the initial time $t_{1}$ (or equivalently, when we constantly change concentration). Starting from Eq. (8) (see also the Appendix A, Case 2) we eventually end up with the non-linear solution:

$$
V(t)=\left\{\begin{array}{cc}
V_{0}+\Phi \gamma T t & t<t_{1} \\
V_{0} e^{\Phi p_{1} \cdot\left(t-t_{1}\right)}+\Phi \gamma T t_{1} & t \geq t_{1} .
\end{array}\right.
$$

Our model and derivated expressions anticipated two kinds of solutions, namely linear and non-linear ones. Indeed, the same sorts of behavior occurred in authors' performed experiments. These empirical results fully verified and confirmed the theoretical approach, see Figures 2 and 3. In Figure 2 we see the linear response of elongation to the external perturbation (stimulator/inhibitor applied once at $t=t_{1}=4 \mathrm{~h}$ ) according to the Eq. (9), with the control subtracted. Similarly, in Figure 3 we see the non-linear response of elongation to the external perturbation (stimulator/inhibitor applied first at $t=t_{1}=4 \mathrm{~h}$, and successively increased at subsequent hours) according to the Eq. (10), with the control subtracted, as in the above case.

FINAL

REMARKS

Accepting as a starting point the time-dependent differential Lockhart growth equation we have introduced the important notion (magnitude) of environmental temperature into it. Such temperature-modified growth equation not only satisfactorily describes the existing growth data but through the exactness of obtained results (which occur via very high determination coefficients) it seems to be a proper candidate for general equation describing phenomena connected with the problem of plant cell growth. Moreover, by taking into account the temperature dependent growth experiments, which also include growth stimulators/inhibitors, we perform further investigations and propose the linear/non-linear response theory which incorporate growth data and give a reasonable theoretical description of the action of plant phytohormones or abiotic factors on the plant growth rate. One of the most interesting findings of our model is that based on the simple analytic predictions we are able to draw qualitative and, especially, quantitative conclusions about the cell wall extensibility $\Phi(\tau)$ itself at least at high-temperature region of non-elastic expansion where the Lockhart equation holds. We stress that the authors' performed experiments confirmed, with a high degree of accuracy, the anticipated theoretically phenomenological solutions. Further detailed study of particular experiments in the light of our theory is presently under investigation.

\section{APPENDIX A \\ TEMPERATURE DEPENDENT LOCKHART EQUATION - DETAILED DERIVATION}

NO EXTERNAL INFLUENCE. UNPERTURBED GROWTH - CASE 0

We consider the Lockhart equation (in the non-elastic regime)

$$
\frac{1}{V} \frac{d V}{d t}=\Phi(P-Y)
$$

where we put $\Phi=\Phi(T)=$ const $(\mathrm{t}), Y=Y(T, V) \rightarrow Y=a T / V$, $a>0$ and $P=P(T, V)=n \mathrm{R} T / V, n>0$.

The turgor pressure $Y$ (as we assume in this model) is reciprocal to the volume $V$, and in our approach we consider the hydrostatic pressure $P$ dependence on $T$ and $V$ from the state equation. The expansion coefficient of the cell wall $\Phi(T)$ we assume as constant in time and dependent only on temperature (for further discussion cf. Appendix B). After substituting thus defined $\Phi, Y$ and $P$ into Eq. (11) we receive after multipyling by $V$ and integrating

$$
V_{T}(t)=V_{0}+\underbrace{\Phi(T) \gamma T t}_{\text {slope }}
$$

where we have substituted $\gamma=n \mathrm{R}-a$. The $\gamma$ value can be estimated numerically from the condition that the difference $P-Y$ is of the order of $0.1 \mathrm{MPa}$.The slope in Eq. (12) is a time-independent directional coefficient easy to fit from the experiment. The volume $V_{T}(t)$ parametrized by temperature $T$ demonstrates the linear character which stays in a good agreement with empirical data within accepted model assumptions. Furthermore, the experimental data (the growth in normal conditions) lead us to the additional conclusion: $\gamma>0$.

\section{PERTURBATION PRESENT. LINEAR RESPONSE - CASE 1}

The plant growth stimulator and inhibitor we can represent as additional pressure "switched on" at an instant $t_{1}$

$$
P \rightarrow P+p_{1} \theta\left(t-t_{1}\right)
$$

Here we consider the case $\mathrm{p}_{1}=\mathrm{b}_{1} / V$ and $\mathrm{b}_{1}=$ const. Then

$$
\begin{gathered}
\frac{1}{V} \frac{d V}{d t}=\Phi(T)\left(\gamma \frac{T}{V}+\frac{b_{1}}{V} \theta\left(t-t_{1}\right)\right) \\
\Downarrow \\
V=V_{0}+\Phi(T)\left(\gamma T t+b_{1} \vartheta\left(t-t_{1}\right)\right)
\end{gathered}
$$

where $\vartheta\left(t-t_{1}\right)$ is the primary function (in the weaker sense) of the Heaviside theta function (we assume $\mathrm{c}=0$, according to the continuity condition at $t=t_{1}$ for the volume $V$ )

$$
\vartheta\left(t-t_{1}\right)=\left\{\begin{array}{cc}
c & t<t_{1} \\
t-t_{1}+c & t \geq t_{1}
\end{array}\right.
$$


PERTURBATION PRESENT

NONLINEAR RESPONSE - CASE 2

However, if we yet assume, as in formula (8) $\mathrm{p}_{1}=$ const., then we obtain inhomogeneous differential equation which we can solve by means of the constant variation

$$
\begin{aligned}
\frac{1}{V} \frac{d V}{d t} & =\Phi\left(\gamma \frac{T}{V}+p_{1} \theta\left(t-t_{1}\right)\right) \\
\Downarrow & \\
\frac{d V}{d t} & =\Phi\left(\gamma T+p_{1} V \theta\left(t-t_{1}\right)\right) .
\end{aligned}
$$

First we solve the homogeneous equation

$$
\begin{gathered}
\frac{d V}{d t}=\Phi p_{1} V \theta\left(t-t_{1}\right) \\
\Downarrow \\
V=V_{0} \cdot e^{\Phi p_{1} \vartheta\left(t-t_{1}\right)}
\end{gathered}
$$

where $V_{0}=V\left(t=t_{0}\right)$ and $t_{0}$ is the start of the experiment. Next, we perform variation of the constant $V_{0}$ :

$$
\begin{gathered}
V(t)=V_{0}(t) \cdot e^{\Phi p_{1} \vartheta\left(t-t_{1}\right)} \\
\Downarrow \\
\frac{d V}{d t}=\frac{d V_{0}(t)}{d t} e^{\Phi p_{1} \vartheta\left(t-t_{1}\right)}+V_{0}(t) \cdot \Phi p_{1} \theta\left(t-t_{1}\right) \cdot e^{\Phi p_{1} \vartheta\left(t-t_{1}\right)}
\end{gathered}
$$

By comparing equations (16) and (18) we receive the following solution for $V_{0}$

$$
V_{0}(t)-V_{0}\left(t_{0}\right)=\Phi \gamma T \int e^{-\Phi p_{1} \vartheta\left(t-t_{1}\right)} d t
$$

Now, if we want to find the primary function for $\exp (-$ $\left.\Phi p_{1} \vartheta\left(t-t_{1}\right)\right)$ we need the explicit form of this exponent

$$
\exp \left(-\Phi p_{1} \vartheta\left(t-t_{1}\right)\right)=\left\{\begin{array}{cc}
1 & t<t_{1} \\
e^{-\Phi p_{1} \cdot\left(t-t_{1}\right)} & t \geq t_{1}
\end{array}\right.
$$

We notice that this function is continuous. Consequently its primary function exists (in the common sense) which is also continuous:

$$
\int \exp \left(-\Phi p_{1} \vartheta\left(t-t_{1}\right)\right) d t=\left\{\begin{array}{cr}
t & t<t_{1} \\
-\frac{1}{\Phi p_{1}} e^{-\Phi p_{1}\left(t-t_{1}\right)}+C & t \geq t_{1}
\end{array}\right.
$$

From the continuity condition we receive $\mathrm{C}=t_{1}+1 / \Phi p_{1}$. Eventually we get the "constant" $V_{0}$ which we insert into Eq. (17) and finally obtain the following result

$$
V(t)=\left\{\begin{array}{cc}
V_{0}+\Phi \gamma T t & t<t_{1} \\
V_{0} e^{\Phi p_{1} \cdot\left(t-t_{1}\right)}+\Phi \gamma T t_{1} & t \geq t_{1} .
\end{array}\right.
$$

Also in this case the volume $V(T, t)$ is continuous.

\section{APPENDIX B}

While constructing the model, especially by introducing simplifications, the following two questions arise: (a) Whether the assumption $\gamma(\mathrm{t})=$ const. is valid, and (b) If the premise about the independence on time of the call wall extensibility coefficient $\Phi(t, T)=\Phi(T)$ is acceptable.

The first question raised can be answered twofold. Firstly, as we notice from the experiment (see Fig. 1) the volume $V$ increases of the order of about $10 \%$ of its initial value $\left(V_{0}=12.5 \mathrm{~mm}^{3}\right)$. It means that the number of water (solution) moles filling up the plant cell will also be greater proportionally to (approximately) the same magnitude. The assumption $\gamma(t)=\mathrm{R} n(t)-a(t)=\operatorname{const}(\mathrm{t})$, even though it is not exact, holds, especially because the maximum of $10 \%$ growth occurs only for the optimum temperature. In all remaining cases (non-optimal temperatures) this value is only of about $5 \%$ which is within the very often accepted limits of experimental error (5\%). Accordingly, the assumption that $\gamma(t)=$ const. is valid (this is also the way we put in the role of osmosis into our model) and gives simple and intuitive analytical solutions which is also important from the applicational point of view. This is, however, the main advantage of our model. The simplicity and aesthetics of our solutions, especially in case of external perturbations (action of growth regulators: stimulators/inhibitors), is in agreement with intuition and reflects the reaction of plant cell on external factors.

Whether $\gamma(t)=$ const. is a good approximation one can also think of in a different way. Namely, let us admit the time dependence of water moles in equation $P=n(\mathrm{t}) \mathrm{R} T / V(t)$. Simultaneously we notice that water is almost ideally incompressible what means that its density is constant $n(\mathrm{t}) / V(\mathrm{t})=\operatorname{const}(\mathrm{t})=n_{0} / V_{0}$ (where $n_{0}$ and $V_{0}$ denote the initial values of the number of water moles and of the volume of plant cell, respectively). Thus we receive the following approximated state equation for water

$$
P=\frac{n_{0} R}{V_{0}} T
$$

Since $Y$ is of the same character as $P$ then $Y=a_{0} T, a_{0}(t)=$ const. Then we insert the latter expressions into the Lockhart equation (1):

$$
\frac{1}{V} \frac{d V}{d t}=\Phi\left(\frac{n_{0} R}{V_{0}} T-a_{0} T\right)
$$

where we have denoted $\gamma_{0}=n_{0} \mathrm{R} / V_{0}-a_{0}$. Solution for Eq. (24) reads

$$
V=V_{0} \exp \left(\Phi(T) \gamma_{0} T t\right)
$$

and is the exponent both in time and temperature; $V_{0}$ stands for the initial cell volume. The exponential function can be expanded into the Taylor series which for sufficiently small argument yields $\mathrm{e}^{x} \approx 1+x$. Such approximation is 
valid since the experimental evidence support linear dependence of elongation in time (see Fig. 1). Hence one can write

$$
\exp \left(\Phi(T) \gamma_{0} T t\right) \approx 1+\Phi(T) \gamma_{0} T t
$$

to end up with

$$
V=V_{0}\left(1+\Phi(T) \gamma_{0} T t\right)
$$

Having noticed that $a_{0}=a / V_{0}$ and $\gamma_{0}=\gamma / \mathrm{V}_{0}$ we finally receive

$$
V=V_{0}+\Phi(T) \gamma T t
$$

which is, as we see, the result for the model where the number of moles is approximately constant in time (and consequently the $\gamma$ coefficient). It seems that the above presented twofold derivation of the assumption $\gamma(t)=$ const. is fully justified.

Trying to answer the second question raised at the beginning of the Appendix let us draw our attention to the fact that $\Phi(T)$ can not be (in a broad time range) constant in time since the plant cell do not expand infinitely. In order to retain the dynamic equilibrium its elongation must be inhibited and the growth must cease altogether. Thus $\Phi(T)$ must eventually diminish in time (damping).

\section{LITERATURE CITED}

CLELAND R.E. 1986. The role hormones in wall loosening and plant growth. Aust. J. Plant. Physiol. 13: 93-106.

COSGROVE D.J. 1986. Biophysical control of plant cell growth. Ann. Rev. Plant Physiol. 377: 377-405.

COSGROVE D.J. 1993. How do plant cell walls extend? Plant Physiol. 102: 1-6.

EDELMANN H.G. 1995. Wall extensibility during hypocotyl growth: A hypothesis to explain elastic-induced wall loosening. Physiol. Plant. 95: 296-303.

FOGG G.E. 1975. The growth of plants. The Chaucer Press, ed. Clay R., Great Britain.

HOAGLAND D.R., ARNON D.J. 1950. The water culture method for growing plants without soil. Calif. Agric. Exp. Stat. Circ. 347: 1-32.

KUTSCHERA U. 2000. Cell expansion in plant development. R. Bras. Fisiol. Veg. 12: 65-95.

LOCKHART J.A. 1965. An analysis of irreversible plant cell elongation. J. Theor. Biol. 8: 264-275.

PROSEUS T.E., ZHU G., BOYER J.S. 2000. Turgor, temperature, and the growth of plant cells: using Chara corallina as a model system. J. Exp. Bot. 51: 1481-1494.

TREWAVAS A.J. 1991. How do plant growth substances work? Plant Cell Environ. 14: 1-12.

WRIGHT S.T.C. 1966. Growth and cellular differentiation in the wheat coleoptile (Triticum vulgare). Factors influencing the growth responce to gibberelic acid, kinetin and indole--3--acetic acid. J. Exp. Bot. 17: 165-176. 